\title{
The expansion of a coronal mass ejection within LASCO field of view: some regularities
}

\author{
V. G. Fainshtein \\ Institute of solar-terrestrial physics SB RAS, \\ P.O. Box 291, 664033, Irkutsk, Russia \\ email: vfain@iszf.irk.ru
}

\begin{abstract}
Forty five limb CMEs related with eruptive prominences and/or near-to-limb posteruptive arcades have been tested. It is shown that CMEs can be divided into two groups. The first group includes coronal mass ejections whose " $2 \alpha$ " latitude angular sizes apparent in the plane of the sky remain unchanged within measurement accuracy of several degrees. The second one is formed by CMEs that expand "non-radially", namely, their angular sizes increase by the relative value (10-30)\% up to the position of the ejection front $R_{F}=R_{\alpha m}$ and run to the maximal value $2 \alpha_{m}$ at this distance. It has been found that CMEs of the second type are, on the average, wider, faster and have an outer shell brighter and with higher plasma density for long distances. It is shown that on average $R_{\alpha m}$ increases as $2 \alpha_{m}$ rises.
\end{abstract}

\section{Introduction}

The apparent angular size (we shall designate it as " $2 \alpha$ ") of coronal mass ejections (CME) is its geometrical measure and simultaneously reflects important physical properties of CMEs: mass and kinetic energy. Near to the surface of the Sun the angular size of a significant part of CMEs grows as they move. Thus, according to on-the-ground coronagraphs Mark 3 and Mark $4\left(R \leqslant 2.45(2.9) R_{0}\right)$, such CMEs make up about one third of the total number (Burkepile et al. 2007). Here $R$ is the plane of the sky distance from the center of the solar disk, $R_{0}$ is the radius of the Sun, the numbers in parentheses refer to Mark 4. A number of researchers have arrived at the conclusion that the angular size of moving CMEs may change even at $R>(2.5-3) R_{0}$ (Eselevich \& Filippov 1991, Stockton-Chalk 2002, Yashiro et al. 2004) ("non-radial" in terms of Stockton-Chalk 2002). According to Eselevich \& Filippov (1990), the angular size of many CMEs recorded in the field of view of the SOLWIND coronagraph, increase by a factor of two or more in the first 2 hours of observation. According to Stockton-Chalk (2002), the angular size of most near-equatorial CMEs observed in the LASCO C3 field of view grow as the CME moves on. In the process, the maximum increase in angular sizes CME was $\approx 5^{\circ}-6.5^{\circ}$, in comparison with radial expansion, in the field of one CME "leg", i.e. was rather small. That CMEs with angular sizes increasing with time (LASCO data) do exist was noted in Yashiro et al. (2004). On the other hand, researchers often believe that changes in CME angular sizes in the LASCO field of view are negligible. Thus, there is still no clear idea about CME angular size variations with time at $R>(2.5-3) R_{0}$. This paper relies on LASCO data to examine regularities in the expansion of CMEs related to eruptive prominences and/or post-eruptive arcades on the limb. 


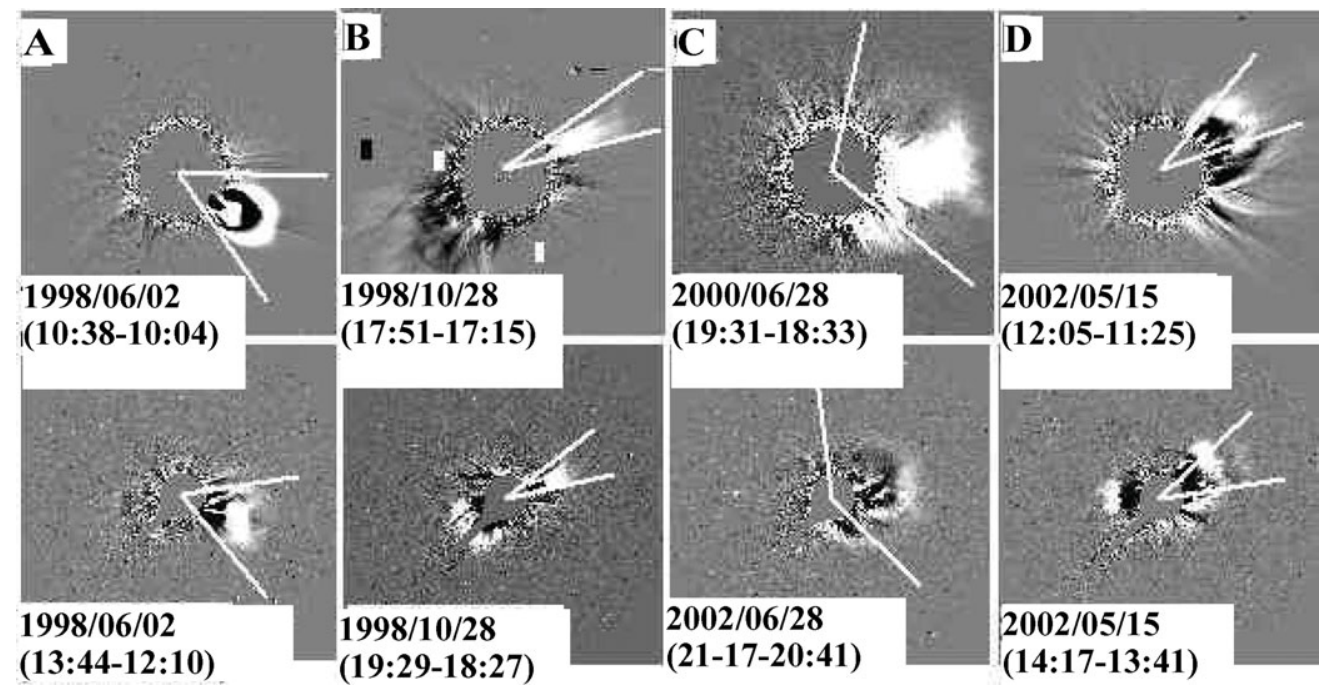

Figure 1. Examples of measuring the angular sizes of CMEs where their boundary determination is hampered by different factors. A and B stands for CMEs with relatively sharp boundaries or with peculiarities at the boundary, which allow the CME angular sizes to be determined within an accuracy of $\approx 3^{\circ}$. C denotes CMEs with a low brightness gradient at the boundary. D means CMEs for which brightness variations of a previous coronal mass ejection near the CME boundary make it difficult to unumbiguously reveal this boundary.

\section{Data and method for analysis}

Two types of SOHO/LASCO data were used to define the CME characteristics: difference images of the corona from the http://cdaw.gsfc.nasa.gov/CME_list/daily_ movies/ database and calibrated coronal images with image-processing level L1.

In most cases, relatively small time/distance variations can be observed in CME angular sizes $2 \alpha$. Therefore, it is necessary to provide for the highest accuracy when deriving $2 \alpha$ values. Although CME angular size has been determined by many investigators, and it is almost a routine procedure, finding these sizes within an accuracy of $2^{\circ}-5^{\circ}$ is rather complicated. The causes of limited accuracy in finding $2 \alpha$ include: (1) small brightness gradient in the CME boundary region; (2) brightness variations of the background plasma or of a previous CME near the CME boundary; (3) insignificantly higher CME brightness near the boundary than the surrounding background brightness. In order to measure the CME sizes (within the above accuracy) we use a time sequence of coronal images to determine the CME boundary; two mutually-supplementary methods are used to find the CME sizes. In the first case, we use differential images to take the $2 \alpha$ value as an angle between two rays drawn from the solar disk center in the plane of the sky to CME boundary features. In the second case, CME angular size is determined using latitude scans of the coronal brightness (calibrated data).

Fig. 1 presents examples of using the first method to derive the $2 \alpha$ value both for cases when it is easy to discern the CME boundary, with much precision (Fig. 1A,B), and for problematic CMEs in terms of their boundaries (Fig. $1 \mathrm{C}-\mathrm{H}$ ). In the first case, the accuracy of deriving $2 \alpha$ may be up to $3^{\circ}-5^{\circ}$. In the second case, the accuracy of measuring CME angular sizes may considerably exceed $5^{\circ}$.

Fig. 2 presents examples of determining CME angular sizes through scanning the brightness of differential calibrated images, at processing level L1. Moreover, in this 

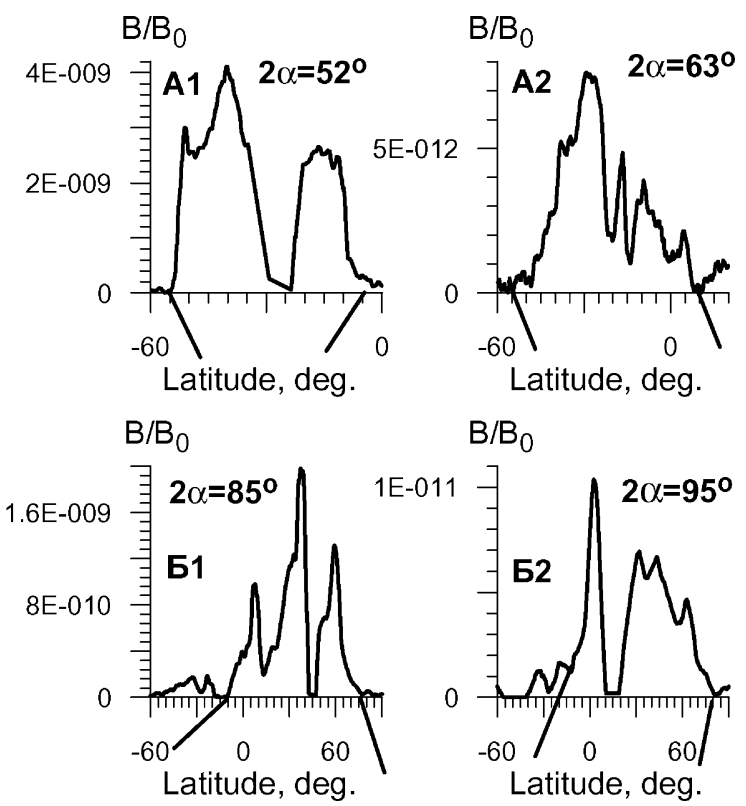

Figure 2. Examples of finding the angular CME sizes using differential scans of calibrated image brightness, at processing level L1. Arrows show CME boundaries. A1-02.06.1998 (10:29-07:02), w-limb, $R=2.75 R_{0} ;$ A2-02.06.1998 (13:44-08:47), w-limb, $R=13 R_{0} ;$. B1-07.01.2001 (5:29-3:53), w-limb, $R=3 R_{0}$; B2-07.01.2001 (9:17-5:17), w-limb, $R=10 R_{0}$. Differences between the moments of time for which the coronal images were subtracted are parathesised near their respective dates. For comparison, Fig. 2 (A1, A2) shows the scans of brightness for two radii (continuous and dotted lines, respectively).

case the brightness was latitude-smoothed, to diminish the high-frequency (noise) signal component.

It is evident from Fig. 2 that in all the above examples the CME boundaries are quite pronounced as places in which the brightness first reaches zero or a minimum value after a strong decrease in the CME body. The angular size is measured accurate to $\approx 3^{\circ}-5^{\circ}$. CME boundary peculiarity is chosen based on an analysis of the time sequence of coronal brightness scans and, when required, a comparison between the scans and the time sequence of CME images. In this paper, a group of 45 limb CMEs, detected in 19972002 and related to near-limb eruptive prominences and/or post-eruptive arcades, were selected for analysis. See the event selection criteria in Fainshtein (2007). The selection of precisely these (limb) CMEs for the analysis is due to the fact that we can determine their true angular sizes. It means that the influence of projective effects should be insignificant.

The EF and PEA characteristics were determined by Sun images in the FeXII $\lambda$ 195A extreme ultraviolet line (SOHO/EIT). The procedure of the EF (PEA) angle size and values $R_{1}$ and $R_{2}$ determination is described in the paper Fainshtein (2007). For analysis we also used the $\beta P-A$ angle position of the EF and/or EPA center (within the heliocentric coordinate system). As V1 values we used this velocity values from the "LASCO CME catalog" (http://cdaw.gsfc.nasa.gov/CME_list/), obtained when linearly approximating the $R_{1}(t)$ points.

\section{Results}

Fig. 3 shows examples of typical CME angular size variations depending on the position of the front $\left(R_{F}\right)$. 


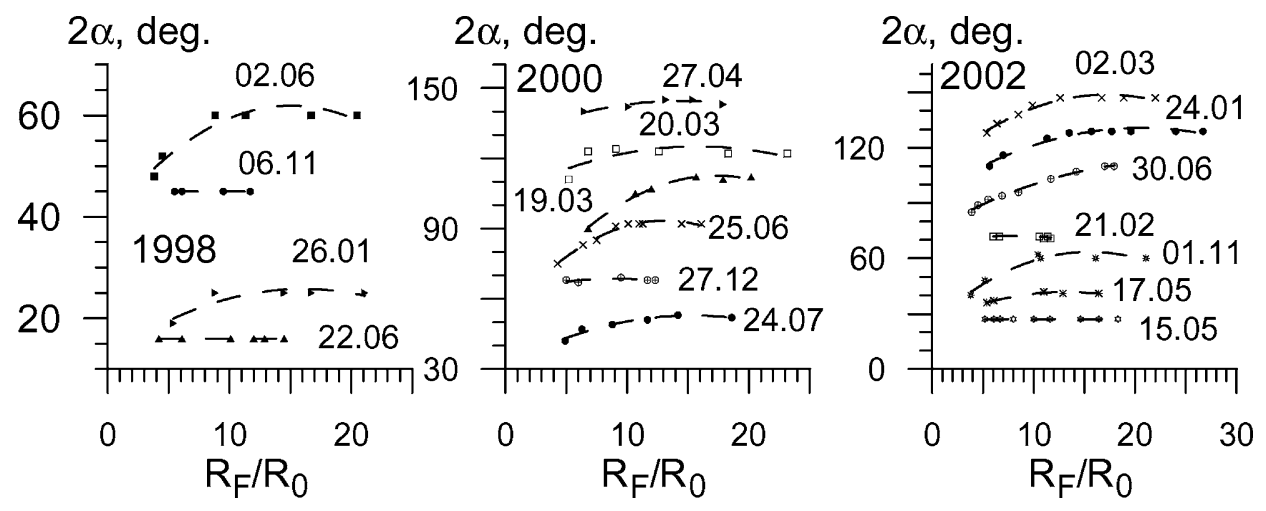

Figure 3. Examples of typical CME angular size variations depending on CME front position $\left(R_{F}\right)$. The numbers near the curves are the CME dates.

The first $2 \alpha$ values were not determined at the moment of CME occurrence, but at the nearest moment when it was possible to determine the CME true angular size. A visual analysis of all dependences $(2 \alpha(R F))$ considered indicates that starting from some $R_{F}$ value (which will be denoted as $R_{\alpha m}$ ), the CME angular size reaches its maximum $2 \alpha_{m}$ value. Further on, this size reaches saturation and becomes stable, or changes insignificantly within an accuracy of $3^{\circ}$, or starts decreasing.

It is established that there is a positive correlation between $R_{\alpha m}$ and $2 \alpha_{m}$, and the connection between these parameters becomes stronger for relatively slow coronal mass ejections (Fig. 4).

Connection between $R_{\alpha m}$ and the speed of the CME front $V_{F}$ (not shown) appears weak and grows for CMEs with rather small angular sizes $R_{\alpha m}$. For the CME velocity $V_{F}$ we take the velocity resulting from the linear approximation of $R_{F}(t)$ from http:// lasco-www. nrl.navy.mil/cmelist.html.

The increase of the CME angular size $2 \alpha$ as the CME moves may be due to different causes. More often, it is related to a faster (in comparison with $2 \alpha=$ const.) increase of transverse linear CME sizes. In some cases, the non-radial CME expansion is associated

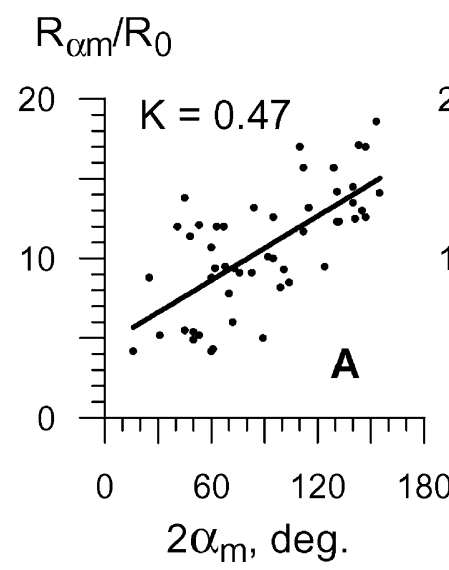

$\mathrm{R}_{\alpha \mathrm{m}} / \mathrm{R}_{0}$

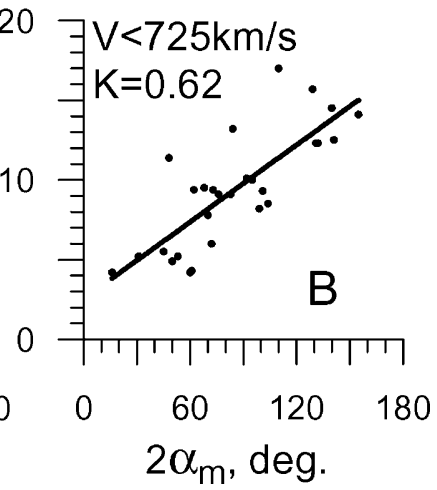

Figure 4. A is the dependence $R_{\alpha_{m}}$ on the maximum CME angular size $\left(2 \alpha_{m}\right)$. B is the dependence $R_{\alpha m}\left(2 \alpha_{m}\right)$ for CMEs with velocities $V_{F}<725 \mathrm{~km} / \mathrm{s}$ (the median velocity value). $K$ is the correlation coefficient. 
with the CME base broadening at the occulter boundary. The third group includes CMEs whose angular sizes increase due to simultaneous action of these two mechanisms. The CME registered on 13 July, 1999 was peculiar. In the course of time, the CME bends and deflects from the radial direction; this results in an apparently strong increase of angular sizes. We used real angular sizes for this CME.

Let us compare some properties of CMEs with radial vs. non-radial expansion. We have selected only those coronal mass ejections into the second group, the increase in whose angular sizes is related to additional expansion due to increased transverse linear sizes. The results of this comparison of the characteristics of the two types of CMEs can be represented using the following figures: the average angular size of radial-expanding CMEs is $64.9^{\circ}\left(29.1^{\circ}\right)$, while that of non-radially expanding ones is $92.4^{\circ}\left(36.8^{\circ}\right)$. The respective mean velocity values are $468(240.9) \mathrm{km} / \mathrm{s}$ and $781(254) \mathrm{km} / \mathrm{s}$. The figures in brackets are the standard deviation.

CMEs of the second type are brighter and denser. Fig. 5 illustrates the higher brightness of the CME outer shell with non-radial expansion. Radial scans of the calibrated brightness of some CMEs are demonstrated here. These scans are drawn along radii near the CME axes. Arrows indicate maximum brightness values in outer CME shells, which we will denote as "CME fronts".

Note that the brightness of the outer part of CMEs with constant angular sizes do not exceed the noise level when the CME front is near the outer boundary of the LASCO C3 field of view $\left(R=(25-30) R_{0}\right)$. At the same time, the brightness of the outer shell of
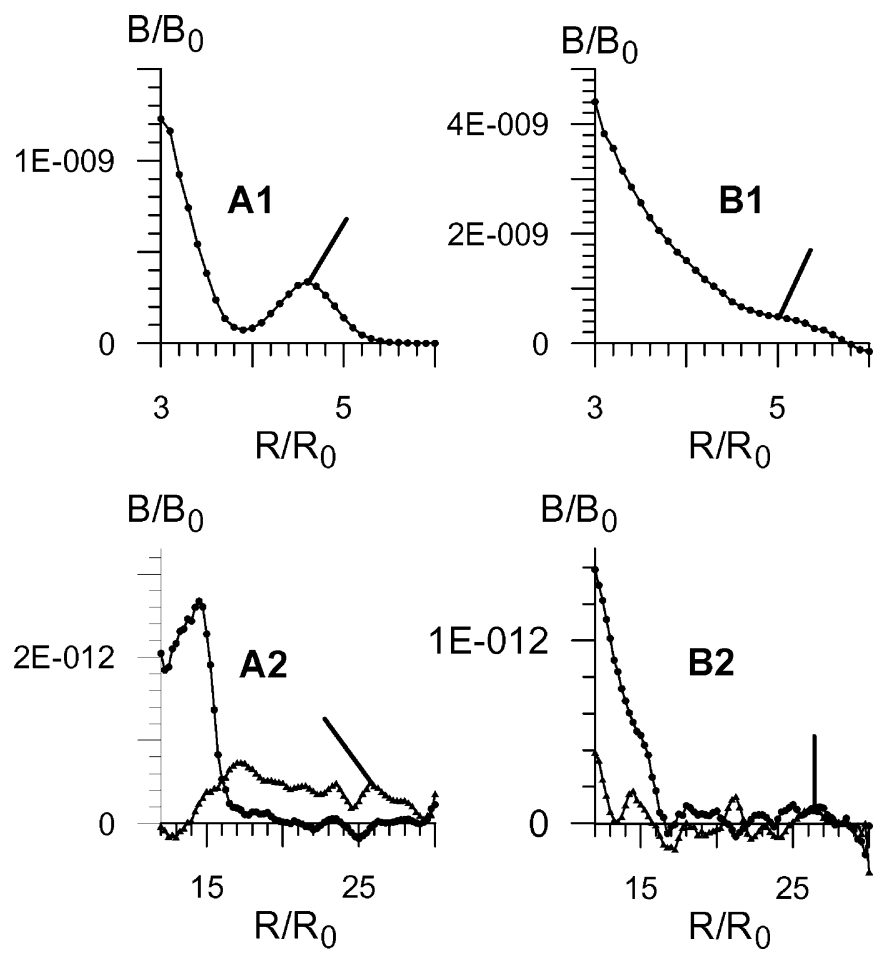

Figure 5. Radial scans of the calibrated brightness for non-radial (A) and radial (B) CMEs. A1: N2, 07.01 .01 (05:29-03:53), A2: N3, 07.01 .01 (09:17-05:17), circles, (13:41-05:17), triangles. B1: N2, 25.11.97 (20:23-19:30), B2: N3, 25.11 .97 (06:42-00:18), circles, (08:42-00:18), triangles. The CME registration dates are accompanied by the differences between the moments for which the coronal images are subtracted from one another. 
non-radially expanding CMEs may exceed the noise level by a factor of $\approx 3$ to $\approx 10$. Fig. 5 proves that, on average, the K-coronal brightness for non-radially expanding CMEs is about 6 times higher than that for radially expanding CMEs; and the angular size of CMEs of the first type is about 1.5 times (see above) as large as the size of the second type. This means that the electron density, and the plasma density, as a whole, in the outer ejection part of CMEs with constant angular size are sometimes lower than in CMEs with non-radial expansion. This suggests that the physical cause of the non-radial CME expansion is increased plasma pressure within the outer CME shell as compared to the background plasma.

In conclusion we shall make one remark. This investigation defined the apparent CME angular size as the size of the angle with its apex at the center of the solar disk, while CME expansion was understood as an increase in this angle. Such a definition of CME angular size is conventional (see (Eselevich \& Filippov 1991, Stockton-Chalk 2002, Yashiro et al. 2004) and the references therein). At the same time the apex of an angle defining the CME size may be placed, basically, at any other spot. There are studies placing this apex, for example, on the surface of the Sun in the CME axis (at the conditional place of CME emergence). It is easy to ascertain that this results in a changed character of the dependence $2 \alpha\left(R_{F}\right)$ in comparison with a case when the apex is located at the center of the solar disk. For example, the angular sizes of CMEs regarded in this work as "radial", will decrease with $R_{F}$. At the same time, it is easy to show that in this case the CMEs in question can also be subdivided into two classes. However, the criteria for assigning CMEs to their proper class will be different. A new class will comprise all those CMEs which had been included into the "radial" group of CMEs, while the other class will acquire the "non-radial" CMEs from this study. This question will be dealt with in more detail in the full version of this work.

Acknowledgements. SOHO is a project of international cooperation between ESA and NASA. This work was supported by RF Leading Scientific Schools Support Governmental Grant SS 4741.2006.2 and by P-16 RAS Presidium Fundamental Research Program.

\section{References}

Burkepile, J. T., St.Cyr, O. C., Stanger, A. L. Sitongia, L., deToma, G., Gilbert, H. \& Darnell, J. A. The presentation at SOHO20, 27-31 August 2007, Ghent, Belgium

Eselevich, V. G. \& Filippov, M. A. 1991 Planet. Space Sci., V. 737

Fainshtein, V. G. 2007, Cosmic Research, 45, 384

Stockton-Chalk, A. 2002 Proc. "SOLSPA: The Second Solar Cycle and Space Weather Euroconference", Vico Equencse, Italy, 24-29 September 2001 (ESA SP-477, February 2002), 277

Yashiro, S., Gopalswamy, N., Michalek, G., St. Cyr, O. C., Plunkett, S. P., Rich, N. B., \& Howard, R. A. 2004 J. Geophys. Res. 109, A07105

\section{Discussion}

HOWARD: I have not seen "non-radial" expansion in the LASCO data except for CMEs that are well out of the plane-of-the-sky. In this case the effect is due to the projection on the $2 \mathrm{D}$ plane.

FAINSHTEIn: I think, for most analyzed CMEs the "non-radial" expansion of the CME is not due to projection onto the plane of the sky. It is a physical effect. But fore some CMEs the "non-radial" expansion possibly may be attributed to projection onto the plane of the sky. 
ERoshenko: Are any of the CMEs full halo or partial halo CMEs?

FAinshtein: No, we have not studied full halo or partial halo CMEs. We have studied "limb" CMEs with axis which is perpendicular to the SunEarth axis.

GIRISH: Have you compared solar wind data for "radial" and "non-radial" CMEs?

FAinshtein: It is hard to do it for CMEs we studied because the CME axis is perpendicular to the Sun-Earth axis. But now such study can be undertaken using data from STEREO. 